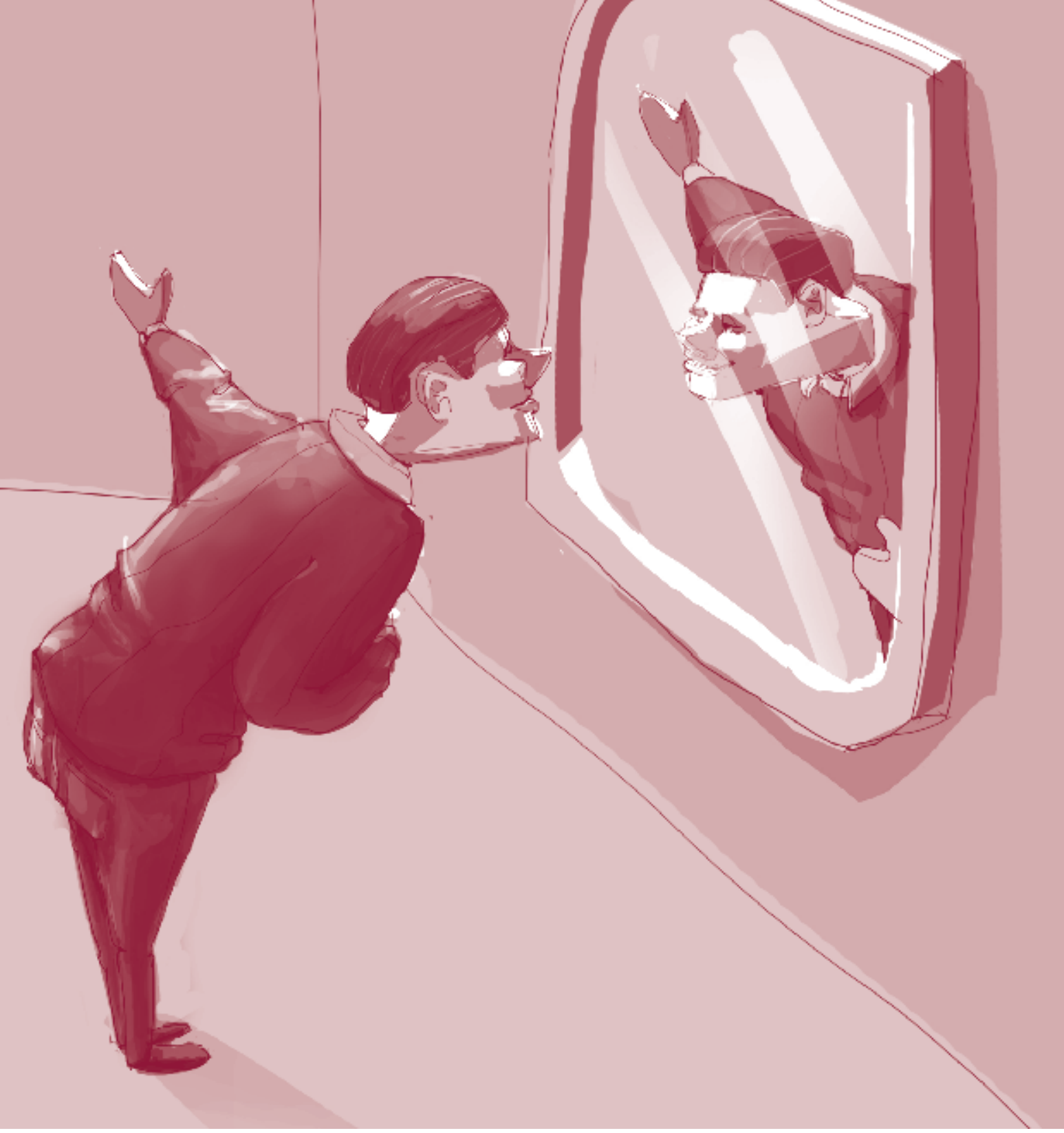

\title{
Hic et nunc: el cuerpo en la educación. Una experiencia de teatro en el aula para el aquí y el ahora
}

Hic et nunc: the body in education. A theatre experience in the classroom for here and now Hic et nunc: o corpo na educação. A experiência de teatro na sala de aula para aqui e agora

Adelaida Corredor Torres 


\section{Adelaida Corredor Torres}

1. Docente de Educación artística-Teatro del Colegio IED República Bolivariana de Venezuela, jornada tarde. Magister en Escrituras Creativas, línea dramaturgia, Universidad Nacional de Colombia. Correo electrónico: acorto2@gmail.com

Fecha de recepción: 29 de octubre de 2014 / fecha de aprobación: 4 de febrero de 2015

\section{Resumen}

Este texto presenta ${ }^{2}$ una experiencia de formación teatral en educación básica y media en un colegio distrital de integración, que buscó establecer la "territorialidad del cuerpo" para promover la dignidad del individuo. La experiencia desarrolla aprendizajes basados en el reconocimiento de sí mismo y el reconocimiento del otro, desde la revisión de modelos de formación no formal. Resultó concluyente que los principios y metodologías asociadas con la educación no formal, pueden generar afianzamiento personal y social al permitir la construcción de vínculos y relaciones relevantes entre "teatro", "cuerpo" y

"educación".

Palabras clave: Cuerpo, teatro, currículo, pedagogía y educación

\section{Summary}

What is presented here is a drama learning experience undertaken in a state primary and middle comprehensive school which sought to establish the "territoriality of the body" in order to promote individual's own dignity. The initiative delivered learning based on self-awareness and the acknowledging of others by reviewing models typical of nonformal education settings. It was concluded that both the principles and methodologies associated with non-formal education can generate personal and social enhancement by allowing the creation of links and building relevant relationships between "theatre", "the body" and "education".

Key words: Body, theatre, curriculum, pedagogy, education

\section{Resumo}

O que aqui se apresenta é uma experiência de aprendizagem de teatro realizada em uma escola pública de integração (primária e secundária) que pretendia estabelecer a "territorialidade do corpo", para promover a dignidade do indivíduo. A experiência desenvolve aprendizagem basados no reconhecimento de si mesmo e do reconhecimento do outro, desde a revisão de modelos de formação não-formal. A conclusão foi que os princípios e metodologias associadas à educação não-formal pode gerar afiançamento pessoal e social ao permitir a construção de vínculos entre "teatro", "corpo" e "educação".

Palavras chave: Corpo, teatro, currículo, pedagogia, educação 
Desde mi cuerpo yo tengo mi cuerpo como extraño para mí, expropiado. El cuerpo es el extraño "allá lejos" (es el lugar de todo extraño) puesto que está aquí. Aquí en el "allá” del aquí, el cuerpo abre, corta, separa el "allá” lejos (Nancy, 2003, p. 18).

n 2010, en una institución educativa distrital ${ }^{3}$ ubicada en — el barrio Santafé, localidad 14 - Los Mártires ${ }^{4}$ en el centro de Bogotá, se da inicio a una experiencia de educación artística fundamentada en el teatro, que permite pensar el cuerpo en el aula, partiendo del reconocimiento del contexto en el que se ubican, tanto el colegio como sus estudiantes, y del rol de docente en un colegio cuyo proyecto educativo institucional (PEI) contiene el ejercicio de los derechos y la dignidad humana, mediante el sistema de inclusión de niños, niñas y jóvenes diagnosticados con Trastorno del Espectro Autista (TEA) y niños, niñas y jóvenes con déficit cognitivo, en el aula regular.

Para su diseño e implementación se recurrió a la revisión de la propia experiencia en prácticas pedagógicas, desarrolladas durante más de veinte años, en proyectos de educación no formal, entre ellos, Talentos artísticos juveniles ${ }^{5}$, Jóvenes tejedores de sociedad ${ }^{6} \mathrm{y}$ Escuelas locales de Formación artística ${ }^{7}$ en Bogotá, originados en la institucionalidad del sector cultura al margen del sector educativo, con diferentes intencionalidades. Tras la revisión se encontró que, en medio de sus diferencias de enfoques y de objetivos, estos proyectos tuvieron en común algunos elementos dignos de ser traídos al aula de bachillerato para verificar su validez y pertinencia en el marco de la educación formal. De esta manera, la experiencia en el colegio inicia con el diseño de un modelo de proceso pedagógico que nace con el análisis de las experiencias citadas anteriormente y deriva en un ambiente de aprendizaje ampliado: el festival artístico que se realiza al finalizar el segundo semestre de cada año, como un espacio dedicado a la socialización de escenificaciones ligadas al teatro, a la danza y a la música, construidas desde el goce de los descubrimientos que hacen las y los estudiantes de su cuerpo como territorio extensible.

Esta experiencia en Educación Artística fue la primera de la jornada de la tarde; como área obligatoria y fundamental del conocimiento, instala el teatro en dos planos simultáneos: uno, como objeto de conocimiento, y otro, como mediación para el encuentro de las y los estudiantes con su corporeidad. A partir de esta ubicación binaria, en el plano del objeto de conocimiento se previeron tres objetos de aprendizaje: actuación, escenotécnicas y dramaturgia, situando la puesta en escena como metodología para el aprendizaje desde la experimentación continua, y, la formulación de logros como una herramienta flexible que permitiera medir los desempeños en estos tres aspectos. Y en el plano de la mediación, los objetos de aprendizaje se concibieron como dominios: esquema e imagen corporal en lo relativo a propiocepción y exterocepción, así como vida cotidiana, gustos, apetencias, repulsiones, proyecciones, familia, historia personal, historia colectiva. A lo largo de estos cuatro años, se verificó que cada grupo de estudiantes tiene especificidades que obligan a repensar los niveles de complejidad de cada logro para cada ejercicio realizado, y, que los dominios permitieron definir diferentes dimensiones de la corporeidad: física, emocional, racional, histórica y ética, además éstos se emplearon como materia prima para las experiencias escénicas al interior del aula.

La literatura que acompañó el análisis de las prácticas en educación no formal permitió precisar el punto de partida para el diseño del proyecto en la comprensión del cuerpo como la existencia, en sus múltiples dimensiones, en un pasado y un futuro que se fusionan en el presente continuo del aquí y el ahora.

3 Colegio IED República Bolivariana de Venezuela, sede A, jornada tarde.

4 El barrio Santafé de la Localidad de los Mártires está ubicado en una zona de alto impacto, término adoptado por el Plan de Ordenamiento Territorial de la Administración Distrital para designar las antes llamadas Zonas de Tolerancia, caracterizadas por sostener la prostitución como una de sus principales actividades económicas, según el Diagnóstico socioeconómico realizado por Hospital Centro Oriente en 2012.

5 Proyecto de Formación Artística implementado por la Administración Nacional desde la Red de Solidaridad Social y el Sistema de selección de beneficiarios de la política social (SIBEN), dirigida a jóvenes entre los 12 y los 25 años de estrato socioeconómico 1 y 2 en varios municipios de Colombia, durante los años 1995 , 1996 y 1997.

6 Escuela de Formación Artística implementada por la Administración Distrital en las 20 localidades de Bogotá, en diferentes disciplinas artísticas en el nivel no formal, como una mirada a la formación artística desde lo social, dirigida a jóvenes entre los 14 y los 25 años de edad, durante los años 1999 a 2004.

7 Proyectos de Formación Artística implementados por algunas administraciones locales en el Distrito Capital. Estas escuelas inician con la organización del Sistema Distrital de Cultura y actualmente se mantienen. 


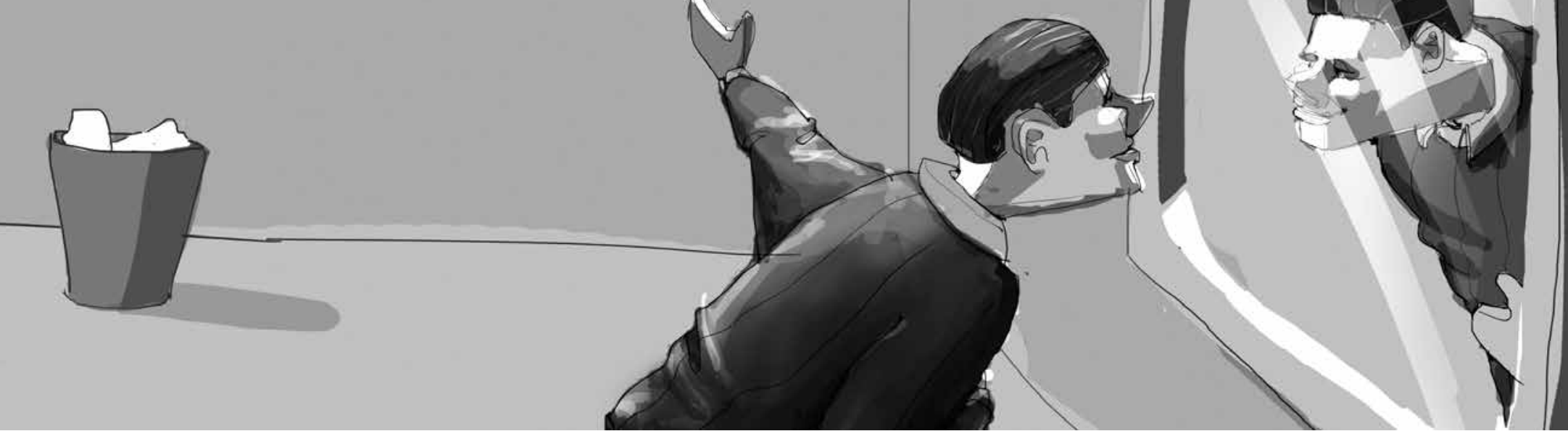

Las condiciones contextuales del colegio y de sus estudiantes impulsaron a considerar la apropiación de la corporeidad como un imperativo de formación. Inicialmente, se encontró que el contexto en el que viven nuestros y nuestras estudiantes determina conductas de supervivencia, evidenciadas en múltiples violencias veladas y explícitas, que generan un estado permanente de vulnerabilidad y riesgo. Y estas conductas cada vez que son escenificadas son también resignificadas permitiendo, a cada estudiante, organizar una nueva vista de su corporeidad, lo que comporta un sentido de apropiación de sí. El aquí y el ahora de la representación teatral renueva la particular manera de existir de cada uno de ellos. Así, las lecturas sumadas a la observación, tanto de los talleres informales como del trabajo en aula, dieron pie a que el cuerpo se instalara como centro del currículo para el área de Educación Artística con una perspectiva de territorio.

Fueron diferentes enfoques los que confluyeron en la construcción del concepto territorio como atributo del cuerpo. En primer lugar, se tuvo en cuenta que el cuerpo debe ser visto, estudiado e interpretado desde múltiples dimensiones (Rico Bovio, 1998) y por esta condición multidimensional, éste no es exclusivamente el entramado de músculos, órganos, huesos y tendones junto con sus posibilidades kinéticas, el cuerpo es también su conocimiento y representación simbólica (Le Boulch, 2001); el cuerpo es el reflejo de toda situación emocional y las emociones son susceptibles de ser orientadas e incluso reorganizadas, dependiendo del desarrollo de la inteligencia emocional de cada sujeto, hasta llegar a expandirse como un cerebro social (Goleman, 2011); el cuerpo también es susceptible de expandirse como sujeto histórico a partir del desarrollo de la voluntad de conocer (Zemelman, 2005). Pero si todo ello en su conjunto es aceptado, entonces, el cuerpo se organiza no solamente en dimensiones, sino también a partir de las relaciones que se establecen entre ellas en tiempo y espacio, y esto nos lleva a afirmar que el cuerpo es la existencia misma (Nancy, 2003). Y la existencia de nuestras y nuestros estudiantes deviene en un contexto rodeado de violencias naturalizadas por la cercanía de una "zona de alto impacto".

En segundo lugar, obedeciendo al impulso de ubicar contenidos y metodologías que respondan a paradigmas educativos del aquí y del ahora, para configurar un ambiente favorecedor de la construcción de relaciones de convivencia empática, basada en la comprensión de las dinámicas propias, individuales, así como de las dinámicas de los otros, fue indispensable revisar los principios que deberían orientar un currículo del cuerpo. Se estableció un marco de acción con del diseño de un modelo de proceso pedagógico y de un ambiente de aprendizaje que concretara aquellos elementos fundantes de las citadas prácticas en educación no formal, desde su revisión y se llegó al reconocimiento de tres principios: integridad, integralidad e interdisciplinariedad. Se estableció que: la integridad entendida como la imposibilidad de separar las dimensiones de la corporeidad en compartimientos únicos, al verificar que todas ellas mantienen una relación sistémica; la integralidad asumida como la capacidad de construir vínculos y relaciones relevantes entre el

8 Concepto tomado del Diagnostico local con participación social, 2012. 
conocimiento y las dimensiones de la corporeidad, y, la interdisciplinariedad empleada como el tránsito entre diferentes disciplinas artísticas para la experimentación en escena, sustentaban aquellas prácticas que en su momento se llamaron talleres de formación teatral. Esta orientación posibilitó en cada una de las experiencias, el encuentro con múltiples y variadas respuestas a un mismo interrogante, y desde luego, el interrogante atendería a unos conceptos y a unas habilidades derivadas de las disciplinas artísticas que el estudiante debería tener presentes para curiosear, escudrinar y descubrir, sus propias dimensiones.

Otro elemento de base, reconocido en los talleres informales, fue la concepción de grupo como sujeto de aprendizaje. El teatro requiere de un cuerpo-grupo para lograr su "vida en el escenario", su existencia frente al público, y de esta reflexión deriva otro componente pedagógico: el sujeto de aprendizaje es el grupo, porque en su seno se construyen y se concertan las reglas de juego, y es también en su seno donde se validan. Es allí en ese cuerpo-grupo donde se experimenta, se reinventa, se estructura una nueva imagen; por ello se concibe el grupo como "territorio extendido" de cada uno de sus integrantes, pues "territorio corporal" es ese lugar donde ocurren los conocimientos y desde el cual se toman las decisiones sobre los aprendizajes construidos y sobre la relación que estos tendrán con su historia personal y con su entorno. El trabajo cooperativo y el trabajo colaborativo permitieron establecer relaciones empáticas y de equidad entre estudiantes regulares y estudiantes integrados, a partir del reconocimiento de los alcances y las habilidades diferenciados. La apropiación de las condiciones individuales y el respeto por las condiciones del otro, en cada ejercicio de representación, permitieron entender el error como una oportunidad de aprendizaje desmontando la idea de fracaso. Cada estudiante fue progresivamente alejado de la noción de equivocación y de la frustración que nace de ella, y esta distancia se originó al interior de grupos heterogéneos que validaron la diferencia como una necesidad para la existencia del teatro y como una condición natural del ser humano.

Pero, ¿qué estilo de "vida en el escenario" sería coherente con los principios y metodologías presentadas? Se toma la función social del teatro para extenderla a la educación: "porque las representaciones deben dar paso a lo representado, a la convivencia de los hombres, y el placer ante su perfección debe ser realzado por el placer, aún más alto, de ver que las normas de la convivencia humana aquí expuestas son tratadas como pasajeras e imperfectas" (Brecht, 1948 p. 19); esta extensión de la premisa brechtiana permite encontrar un vínculo directo entre diversión en la experimentación escénica y re-significación de las situaciones cotidianas en el aula. Extensión que orientó la revisión de conceptos pedagógicos que han tomado distancia de la escuela tradicional y que han reubicado el rol del docente.

De un lado, las invariantes pedagógicas de la escuela moderna (Freinet, 1960) nos indican que el éxito educa más que el fracaso; y de otro lado, la educación en segunda persona (Not, 1992) invita a establecer un diálogo respetuoso entre los contenidos, el estudiante y el docente. Estas dos perspectivas se enmarcan en el cuestionamiento de los contenidos de la escuela tradicional en torno a la enseñanza de materias desarticuladas y descontextualizadas "[...] que cuando el estudiante termina sus estudios, los conocimientos supuestamente adquiridos ya no le sirven para nada práctico en la vida" (Zuleta, 2004, p.15).

Si bien, durante la fase de observación y diagnóstico de la situación, a mediados de 2010, se evidenció que la instalación del teatro como asignatura plantea un espacio de incertidumbre para los y las estudiantes, frente al cual se activan sus mecanismos de defensa, gracias a lo cual se verificó que la actitud de niñas, niños y jóvenes dio un giro favorable. Lo anterior se hizo mediante una encuesta de percepción, aplicada en 2013 a una muestra representativa de estudiantes de los grados $6^{\circ}$ a $11^{\circ}$, en el marco del proyecto Arte y Corporeidad en la Escuela. ${ }^{9}$

En primera instancia, el teatro no es un producto mediático, tal como las prácticas de algunos géneros dancísticos o musicales, y por tanto, no está instalado en el imaginario de los estudiantes como un bien cultural de uso cotidiano; es decir, ese espacio y tiempo en el que se desarrollan rutinas de trabajo corporal, representación de situaciones y de re-lectura de su contexto de una manera crítica, divertida y creativa es un territorio desconocido, agobiante, y posiblemente inservible, cuya incompatibilidad con su corporeidad se declaró mediante actitudes negativas hacia las propuestas de trabajo. En segunda

9 Arte y Corporeidad en la Escuela es un proyecto iniciado en 2013, como parte de la metodología para el estudio sobre saberes y mediaciones circulantes en experiencias significativas en la temática "arte y corporeidad" en instituciones educativas distritales, liderado por el IDEP y ejecutado por la Universidad Javeriana. 
instancia, durante este primer semestre se observó en un gran número de estudiantes la naturalización de prácticas violentas en su cotidianeidad, tales como el uso constante de palabras soeces, la intimidación, la negación del otro, la burla frente a la diferencia, la imposición por la fuerza, la indiferencia frente al dolor ajeno y la autoagresión, sumadas a la resistencia a la autoridad. La composición diversa de la población del barrio Santafé, las condiciones de insatisfacción de las necesidades básicas marcadas por el hacinamiento, la violencia doméstica, la presencia de bandas juveniles y delincuencia común, organizan un territorio apto para el desencanto, la desmotivación y, por consiguiente, configuran una serie de dificultades para el desarrollo de un proyecto que busque potenciar condiciones de dignidad para la propia existencia, y de manera ambivalente estas dificultades estimulan su implementación.

Sin embargo, a finales de 2013 se constató un cambio evidente en la mirada que los estudiantes tienen de sí mismos y de lo que ellos llaman la "clase de teatro". Los estudiantes registran nuevos conocimientos acerca de sí mismos, descubren sus potencialidades para el canto, la danza folclórica, la improvisación y desde allí, se reivindica el colegio como un espacio para el disfrute a partir de la representación artística con el II Festival Artístico que dedicó tres días a la visibilización de las experiencias artísticas de los estudiantes. Tanto en las muestras finales como en los ensayos y en la preparación de las mismas, la integración de estudiantes con capacidades diferenciales (TEA y Déficit Cognitivo) se da de una forma espontánea y respetuosa; se agudiza el sentido de la escucha y la apreciación de las producciones de los otros, permitiendo validar la diferencia como un aporte a la creatividad.

La aplicación de la encuesta de percepción permitió reconocer a qué le dan valor en el proyecto. Los y las estudiantes dejan ver cómo sus expectativas han ido cambiando, y enuncian la "clase de teatro" como algo necesario para su bienestar, importante para el desarrollo de habilidades en un espacio disfrutable $\mathrm{y}$, en algunos casos, determinante para su proyecto de vida. La importancia que le dan tanto a la representación escénica y a la creatividad manifiesta en la improvisación, como a los aprendizajes socio-emocionales tales como la empatía, la autorregulación, el autocuidado y la ampliación de la conciencia corporal, hacen evidente el redimensionamiento de su territorio desde la aceptación de su propio cuerpo y desde la integración natural a sus experiencias artísticas, de las personas que consideran diferentes. Del espacio de agobio e incertidumbre se ha pasado a un territorio seguro y necesario, tal como se demuestra en las afirmaciones de los estudiantes de las cuales se citan algunas:

Diego Alejandro, de $10^{\circ}$ grado: "[la clase de teatro] Es una de las más importantes del colegio aunque unos la vean como insignificante, pero es la clase donde se dan a conocer los grandes talentos de las personas".

Ricardo, estudiante de $10^{\circ}$ grado afirma: "yo pienso que el arte de aplicar lo que he aprendido a la vida real [es el aprendizaje más importante]".

Tania, estudiante de $8^{\circ}$ : "puedo hacer y descubrir cosas de mí misma (virtudes) que no sabía que podía”.

Lina, estudiante de $10^{\circ}$ : "El teatro para mí significa pasión, mi mayor alegría, mi gran sueño, es arte, es vida, es el mejor modo de expresarnos... no es suficiente con un papel y un esfero para describir lo que es el teatro en mi vida".

Miguel Ángel de 6: lo importante de la clase de teatro es: "hacer las cosas con el corazón para que nos vaya bien".

Estas afirmaciones de estudiantes permiten ver que el tejido organizado entre cuerpo, teatro y pedagogía enunciado como extensión del territorio corporal, ha tenido incidencia en el territorio individual al permitir que el cuerpo piense acerca de sí mismo desde diferentes dimensiones: histórica, ética, física, racional, emocional enmarcadas en un contexto cultural definido. He aquí un territorio extendido más allá del canon, más allá del error, más allá de sí. Sin embargo, este ensanchamiento demostró una fragilidad, pues el sentido de la diferencia para nuestras y nuestros estudiantes emerge de imaginarios colectivos propios del contexto violento y de imaginarios mediáticos y estandarizantes, que compiten a diario con la posibilidad de incluir prácticas diferenciadas y condiciones diferenciales en el aula, lo que hace que las otredades se conciban como erróneas; esta concepción genera un retorno al inicio del proyecto con cada ingreso de un nuevo estudiante. Volvemos al comienzo: al aquí y ahora. Entonces, depende de la dinámica propia de cada grupo, que ese retorno sea comprendido como un afianzamiento o como un retroceso en el proceso de autoreconocimiento y de reconocimiento del otro y de lo otro. Esta fragilidad obliga a replantear, de forma constante, los métodos de evaluación, invitando al fortalecimiento de la autoevaluación, como medio para afianzar las extensiones individuales alcanzadas; a consolidar la co-evaluación, como medio para respaldar las extensiones grupales, y a reforzar la heteroevaluación, como mecanismo de distanciamiento de los estándares mediáticos, para extender las fronteras de cada 
territorio. Y es en esta búsqueda permanente de principios, contenidos y metodologías que impulsan una intencionalidad en la construcción del conocimiento, donde se renueva el rol del docente como investigador de su propia práctica, a la vez que lo afirma como cuerpo territorio, para "Que se escriba no del cuerpo, sino el cuerpo mismo” (Nancy, 2003, p. 11) .

\section{Referencias}

Alcaldía Mayor de Bogotá-Hospital Centro-Oriente. (2012) Diagnóstico Local con participación social, 2012. http://www.esecentrooriente.gov. $\mathrm{co} / \mathrm{hco} /$ images/stories/pic/Epidemiologia/dxlocal/Diagnostico $\% 20$ Local $\% 202012 /$ martires/DIAGNOSTICO $\% 20$ LOCALIDAD $\% 20 L O S \% 20$ MARTIRES $\% 20$ CAPITULO $\% 20 I, \% 20 I I, \% 20$ III\%20,IV\%202012.pdf. Recuperado el 20 de 02 de 2013.

Brecht, B. (s.f.). El pequeño Organon para el teatro escrito en 1948. Recuperado el 24 de octubre de 2014, de http:/ / www.ipprojazz.cl/intranet profesor/subir_archivo/archivos_subidos/24993807-Brecht-Bertolt-Pequeno-organon-para-el-teatro-completo-1948[1].pdf

Freinet, C. (1960). Las invariantes pedagógicas: 30 proncipios de práctica educativa. (C. Armengol, Productor, \& Libros Recuperados. Educación Social). Recuperado el 24 de octubre de 2014, de http:/ /www.raco. cat/index.php/EducacioSocial/article/viewFile/178826/242014

Goleman, D. (2011). El cerebro y la inteligencia emocional: nuevos descubrimiento. (C. Mayor, Trad.) Barcelona, España: Ediciones B, S.A.

Le Boulch, J. (2001). El cuerpo en la escuela del siglo XXI. Primera edición. (I. P. Casellas, Trad.) Barcelona, España: Inde Publicaciones.
Makarenko, A. (1933 [2009]). Poema pedagógico, Primera parte pdf. (frentepopular.files.wordpress.com) Recuperado el 20 de octubre de 2014, de https:// frentepopular.files.wordpress.com/2009/11/poemapedagogico.pdf.

Ministerio de Educación Nacional. Dirección General de Investigación y Desarrollo Pedagógico. Grupo de Investigación y Pedagogía. (2000). Educación artística. Lineamientos curriculares. Bogotá: Editorial M. d. Nacional.

Nancy, J.-L. (2003). Corpus (2 ed.). (P. Bulnes, Trad.) Madrid, España: Arena Libros.

Not, L. (1992). La enseñanza dialogante. Hacia una educación en segunda persona. Barcelona, España: Herder.

Rico Bovio, A. (1998). Las fronteras del cuerpo. Crítica de la corporeidad $\left(2^{\circ}\right.$ ed.). Quito, Ecuador: Ediciones Abya-Yala.

Zemelman, H. (2005). Voluntad de conocer. El sujeto y su pensamiento enel paradigma crítico. (1a ed.). Barcelona, España: Ántrhopos.

Zuleta, E. (2004). Educación y democracia. Un campo de combate (6a ed.). Medellín, Colombia: Hombre Nuevo Editores. 


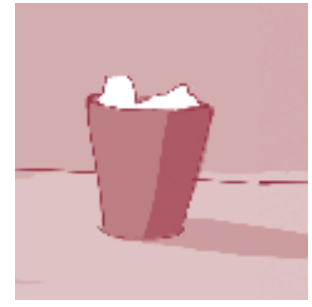

